\title{
Increased plasma malondialdehyde associated with cerebellar structural defects
}

\author{
V Th Ramaekers, B Bosman, G A Jansen, R J A Wanders
}

\begin{abstract}
Background-Malondialdehyde (MDA) in plasma is regarded as an indicator for increased lipid peroxidation.

Method-Measurements of MDA concentrations in plasma were compared among healthy children $(n=31)$, patients with neurological disorders or epileptic syndromes $(n=15)$, and children with pontocerebellar structural defects $(n=31)$, where the cause or genetic defect remained unknown.

Results-In healthy children the median MDA value was $5.86 \mathrm{nmol} / \mathrm{ml}$ (mean (SD) value: 6.25 (1.97), range: 3.76-11.19). For the group with various neurological disorders or epilepsy, the values were similar with the median value at $5.66 \mathrm{nmol} / \mathrm{ml}$ (range 0.22-10.86). Compared with healthy controls and the neurological/ epileptic group, the 31 children with pontocerebellar structural defects had significantly increased MDA values with a median value at $11.29 \mathrm{nmol} / \mathrm{ml}$ (mean (SD) value: 11.62 (3.27), range 3.65-19.22). Implication-These findings of increased plasma MDA in the majority of children with pontocerebellar structural defects of unknown origin raised the question whether increased lipid peroxidation leads to prenatal and postnatal pontocerebellar maldevelopment or degeneration. (Arch Dis Child 1997;77:231-234)
\end{abstract}

Keywords: malondialdehyde; lipid peroxidation; cerebellum

Department of Paediatrics, University Hospital Aachen, Germany V Th Ramaekers

B Bosman

Department of Paediatrics and Clinical

Biochemistry, University Hospital Amsterdam, The Netherlands G A Jansen R J A Wanders

Correspondence to: Dr V Th Ramaekers Department of Paediatrics, Medizinische Einrichtungen der RWTH,

Pauwelsstrasse 30

D-52057 Aachen,

Germany.

Accepted 23 April 1997

Oxidative stress is defined as oxygen radical mediated damage to biological material (proteins, lipids, carbohydrates, and DNA) caused by either increased generation and build up of the oxygen radicals (superoxide radical, hydrogen peroxide, and the hydroxyl radical), or due to the diminished removal or inadequate protection against these ubiquitously present oxygen radicals. During evolution of aerobic life, powerful antioxidant enzymes and radical scavengers have developed to remove or scavenge these oxygen radicals in order to protect cell organelles and membranes. ${ }^{1-4}$

In experiments on cerebellar development in the mouse, gamma irradiation and several toxins are known exogenous agents to generate increased free radicals setting off a mechanism of programmed cell death or apoptosis in cerebellar granule cells. Early apoptosis induced by these exogenous factors results in cerebellar hypoplasia, as cerebellar granule cells are responsible for the bulk of volume growth in the developing cerebellum. ${ }^{5}$ Increased generation of reactive oxygen species and lipid peroxidation followed by apoptosis has also been shown recently in cultures of cortical neurons from fetal brains with Down's syndrome. Degeneration of Down's syndrome neurons could be prevented by treatment with free radical scavengers or catalase. ${ }^{6}$ The known moderate cerebellar hypoplasia in patients with Down's syndrome might reflect the effect of early degeneration of cerebellar progenitor cells by increased lipid peroxidation and apoptosis in vivo. Progressive cerebellar hypoplasia or atrophy has also been found to be a prominent finding in some well defined mitochondrial diseases of the central nervous system where increased oxidative stress might play an important part in the pathogenesis of these disorders. ${ }^{248}$ Examples of these conditions included mitochondrial diseases such as Kearns-Sayre syndrome, myoclonic epilepsy with ragged red fibres (MERRF) syndrome, and cytochrome $c$ oxidase deficiency. Owing to the block of electron flow alongside the respiratory chain enzymes, electrons will leak and produce more superoxide radicals. The consequent production of hydroxyl radical and other powerful radicals can initiate a chain reaction of lipid peroxidation in which polyunsaturated fatty acid side chains are converted into lipid peroxides. One of the end products of lipid peroxidation is malondialdehyde (MDA).

Based on these observations, we decided to screen patients with pontocerebellar maldevelopment or degeneration for MDA as an indicator of increased lipid peroxidation.

\section{Patients and methods}

\section{DESCRIPTION OF PATIENTS}

A total of 31 healthy children without a previous history of any birth trauma, neurological disorder, or epilepsy served as a reference group for plasma MDA values. The median, mean value, and SD have been calculated. The median age was 7.2 years with a range between 1-19.

MDA values were also determined in a second group of 15 children who were known to suffer from various neurological disorders or epileptic syndromes and in whom neuroimaging had excluded infratentorial structural abnormalities. Their median age was 3 years with a range between $0.8-12$ years. In all children, extensive investigations excluded inborn errors of metabolism, mitochondrial, lysosomal, or peroxysomal disorders. Depending on whether frequent daily fits occurred or 
Table 1 The diagnosis is listed for the different groups of neurological patients without $(n=15)$ and with pontocerebellar involvement $(n=31)$

\begin{tabular}{|c|c|c|c|}
\hline Neurological patients with normal infratentorial structure & No & Unilateral and bilateral cerebellar abnormalities & No \\
\hline $\begin{array}{l}\text { Epileptic subgroup: } \\
\text { Infantile epileptic syndromes } \\
\text { Progressive neuronal degeneration of childhood (Alpers' disease) } \\
\text { Occasional fits } \\
\text { Non-epileptic subgroup: } \\
\text { Congenital hemiplegia and pseudo-Lennox syndrome } \\
\text { Landau-Kleffner syndrome and ESES } \\
\text { Mental retardation with choreoatethosis } \\
\text { Volvulus and short bowel syndrome }\end{array}$ & $\begin{array}{l}1 \\
3 \\
2 \\
1\end{array}$ & $\begin{array}{l}\text { Unilateral hypoplasia with psychomotor retardation } \\
\text { Vermis agenesis or midline fusion defects: } \\
\text { Joubert's syndrome } \\
\text { Mental retardation with cap myopathy } \\
\text { Lower vermis agenesis or vermis hypoplasia } \\
\text { Non-progressive cerebellar hypoplasia: } \\
\text { Acrocallosal syndrome } \\
\text { Dandy-Walker syndrome } \\
\text { Dandy-Walker variant } \\
\text { Pontocerebellar hypoplasia or atrophy: } \\
\text { Pontocerebellar hypoplasia type I } \\
\text { Pontocerebellar hypoplasia type II } \\
\text { PEHO syndrome } \\
\text { Microcephaly with pontocerebellar atrophy } \\
\text { Progressive cerebellar degeneration or atrophy: } \\
\text { Infantile and late infantile ceroid lipofuscinosis } \\
\text { Sjögren-Larsson syndrome } \\
\text { Ataxia telangiectasia } \\
\text { Boucher-Neuhäuser syndrome } \\
\text { Spastic ataxic syndrome } \\
\text { Progressive ataxia with polyneuropathy }\end{array}$ & $\begin{array}{l}2 \\
1 \\
1 \\
1 \\
3 \\
2\end{array}$ \\
\hline
\end{tabular}

seizures were only rare events, a further classification distinguished between eight children with epilepsy and seven non-epileptic children. In the group of eight epileptic children, the following clinical entities have been diagnosed: infantile epileptic syndromes with neurodevelopmental retardation in five children, progressive neuronal degeneration with liver disease (Alpers' disease) in one infant, and occasional fits in two children. In the group of seven non-epileptic children, four children had suffered from seizures or epilepsy in the past but all showed an excellent response to anticonvulsant drug treatment. The latter group included congenital hemiplegia and pseudo-Lennox syndrome in one child, Landau-Kleffner syndrome and electrical status epilepticus during slow sleep in three children, mental retardation with choreoathethosis in two children, and short bowel syndrome after jejunoileal resection for volvulus in one child.

The third group of 31 patients displayed structural pontocerebellar abnormalities of heterogeneous aetiology detected by neuroimaging. Their median age was 8 years with a range from 0.6-20 years. Further classification of this group was made depending on the site of the structural abnormality and on the presence of a static or progressive clinical course. Seven patients suffered from psychomotor retardation associated with unilateral cerebellar hypoplasia.

Five patients suffered from midline fusion defects or vermis agenesis. Among the children with vermis agenesis two patients presented a typical history and clinical findings consistent with Joubert's syndrome. The three other children had mental retardation with complete or incomplete vermis fusion defects. In one of the children with incomplete vermis fusion defects, the muscle biopsy specimen was consistent with cap myopathy. Non-progressive cerebellar hypoplasia was detected in three children. One child suffered from the acrocallosal syndrome, one from Dandy-Walker syndrome, and one from the Dandy-Walker variant.

Six children had a syndrome with pontocerebellar hypoplasia or atrophy. One child suffered and died from autosomal recessive pontocerebellar hypoplasia type I, three children from pontocerebellar hypoplasia type II, one child suffered from progressive encephalopathy with hypsarrhythmia and optic atrophy (PEHO), and one from progressive microcephaly with pontocerebellar atrophy.

Ten patients suffered from syndromes associated with progressive degeneration of all cerebellar structures. The established diagnosis included the following disease entities: infantile and late infantile type of neuronal ceroid lipofuscinosis, Sjögren-Larsson syndrome, ataxia telangiectasia, Boucher-Neuhäuser syndrome, two siblings with a progressive spastic ataxic syndrome, one patient with an early onset spastic ataxic syndrome with cerebellar atrophy and dysmyelination, and two patients with progressive ataxia associated with a severe polyneuropathy. Except for sporadic fits in the patient with PEHO syndrome and the two patients with infantile and late infantile ceroid lipofuscinosis, all other patients with cerebellar structural defects did not suffer from convulsions.

Table 1 lists an overview of the clinical or descriptive diagnosis. In most disease entities the genetic or neurometabolic disturbance has not yet been identified.

\section{MEASUREMENT OF MDA}

MDA was measured using the thiobarbituric acid based procedure described by Wasowicz et al. ${ }^{9}$ Briefly, $50 \mu \mathrm{l}$ of plasma are added to 0.95 bidistilled water followed by the addition of 1 $\mathrm{ml}$ of a solution containing $29 \mathrm{mmol} / 1$ thiobarbituric acid in $8.75 \mathrm{~mol} / 1$ acetic acid. Samples are heated for 60 minutes at $95^{\circ} \mathrm{C}$ and cooled down, then $25 \mu \mathrm{l}$ of a solution containing 5 $\mathrm{mol} / \mathrm{l}$ hydrochloric acid is added.

After adding of $3 \mathrm{ml} \mathrm{1-butanol,} \mathrm{samples} \mathrm{are}$ centrifuged $\left(1000 \times g, 10\right.$ minutes, $\left.20^{\circ} \mathrm{C}\right)$ and fluorescence measured (excitation wavelength: $525 \mathrm{~nm}$; emission wavelength: $547 \mathrm{~nm}$ ).

STATISTICAL ANALYSIS AND SD VALUES

For each group the median, mean, and SD values for MDA concentrations have been calculated. For statistical analysis of the data, the 


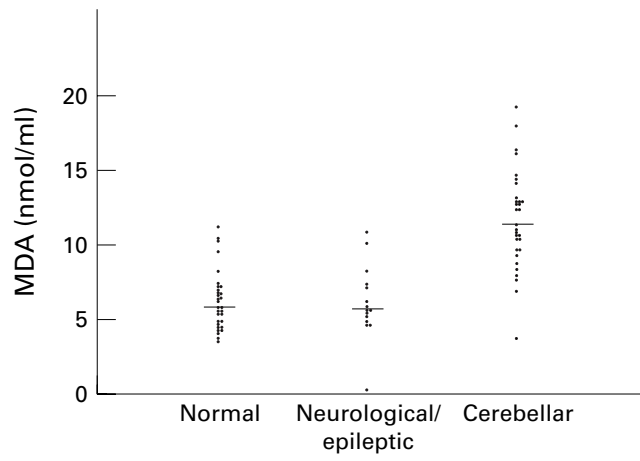

Figure 1 The individual values and the median value (horizontal line) calculated for each group have been plotted. Using the Wilcoxon two sample test no significant differences were present among healthy controls and neurologicallepileptic disorders $(p=0.91)$. A statistically significant difference was found between the group with cerebellar defects in comparison with healthy controls $(p=0.0001)$, or with the neurologicallepileptic group $(p=0.0001)$.

Wilcoxon two sample test was used to test for statistical differences of MDA plasma concentrations among healthy children, the group of neurological/epileptic children, and the group with cerebellar structural defects.

\section{Results}

The results of MDA concentrations in plasma with their respective median values have been plotted for each group (fig 1).

For the control group of 31 healthy children, plasma MDA concentrations showed a mean value of $6.25 \mathrm{nmol} / \mathrm{ml}$ with a SD of 1.97 $\mathrm{nmol} / \mathrm{ml}$ (median value 5.86 ).

In 15 children with various neurological disorders (epilepsy, extrapyramidal disorders, and undernutrition), in whom neuroimaging had excluded cerebellar involvement, the MDA concentrations with a median value at 5.66 $\mathrm{nmol} / \mathrm{ml}$ were situated within a similar range as compared with healthy controls (Wilcoxon two sample test non-significant with $\mathrm{p}=0.91$ ). Within the group of neurological/epileptic disorders, a subdivision between non-epileptic and epileptic children, corresponding to group A1 and A2 in fig 2, was made. Although all MDA concentrations in the non-epileptic subgroup of seven children (group A1) remained below the calculated mean value for the whole group, in the subgroup A2 of eight children with epilepsy MDA values were found to be increased up to $10.8 \mathrm{nmol} / \mathrm{ml}$.

Compared with healthy children and patients with neurological/epileptic syndromes, MDA concentrations for patients with cerebellar structural defects $(n=31)$ were found to be increased significantly (both $\mathrm{p}$ values equal to 0.0001; Wilcoxon two sample test). The median value for the cerebellar group was determined at $11.29 \mathrm{nmol} / \mathrm{ml}$. Figure 2 displays the individual MDA concentrations classified according to each subgroup with specific cerebellar structural defects.

MDA values were constantly increased with values ranging between 9.6 and $16.08 \mathrm{nmol} / \mathrm{ml}$ in seven tested patients (group B) suffering from moderate or severe mental retardation and unilateral cerebellar hypoplasia of undetermined origin.

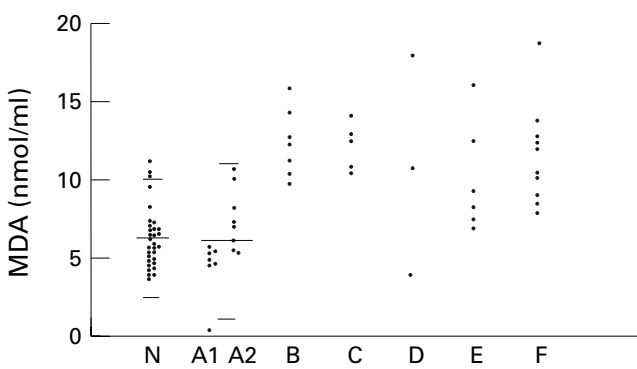

Figure 2 Measured $M D A$ values in $n m o l / m l$ in patients with and without pontocerebellar structural abnormalities; group $N$ stands for normal healthy controls, group $A 1$ and A2 represent patients with normal infratentorial structures, where $A 1$ are patients without seizures and group $A 2$ are patients with epilepsy; group $B$ have unilateral cerebellar hypoplasia; group $C$ have midline vermis fusion defects, group $D$ have non-progressive cerebellar hypoplasia; group $E$ have pontocerebellar hypoplasia; and group $F$ represent patients suffering from progressive cerebellar atrophy. The horizontal lines indicate the mean with upper and lower 2 $S D$ values for $M D A$ in the healthy control group and the neurologicallepileptic group.

MDA values measured in five patients with vermis defects (group C) ranged between 10.55 and $14.25 \mathrm{nmol} / \mathrm{ml}$, that is, in two patients with Joubert's syndrome, one patient with cerebellar ataxia and cap myopathy, another patient with complete vermis agenesis and mental retardation, and one patient with developmental delay after vacuum extraction.

In the group of three patients with nonprogressive and static cerebellar hypoplasia (group D), MDA values were found to be increased in the child with acrocallosal syndrome and the child with the Dandy-Walker variant. In contrast, the MDA value in the child with Dandy-Walker syndrome was normal.

MDA values between 6.96 and 16.28 $\mathrm{nmol} / \mathrm{ml}$ were found in six children belonging to the group of pontocerebellar hypoplasias or atrophy (group E), that is, pontocerebellar hypoplasia type I and II, PEHO syndrome, and the retarded patient with progressive microcephaly and pontocerebellar atrophy. Only two out of six values were increased well above the upper range of normal, whereas the other four MDA values had an overlap with the normal range in healthy individuals.

MDA measurements in 10 patients belonging to the group with progressive bilateral cerebellar atrophy (group F), showed constantly raised values in eight patients. These comprised two children with infantile and late infantile onset neuronal ceroid lipofuscinosis, one child with Sjögren-Larsson syndrome, one with ataxia telangiectasia, one with BoucherNeuhäuser syndrome, one with a spastic ataxic syndrome and dysmyelination, and two patients suffering from progressive cerebellar atrophy combined with a peripheral neuropathy. Intermittent increases were found in the two siblings suffering from an autosomal recessive spastic ataxic syndrome with parkinsonian features. For these two siblings, only the increased MDA values have been plotted in figs 1 and 2 .

In five out of these 10 patients in group $\mathrm{F}$, there was an overlap of the calculated values as compared with the healthy controls. 


\section{Discussion}

In an effort to delineate the unclassified or unknown genetic causes of cerebellar syndromes, MDA values in plasma have been used as a screening procedure for detecting increased lipid peroxidation due to oxidative stress. ${ }^{23}$ Oxidative stress is defined as oxygen radical mediated damage to biological material (proteins, lipids, carbohydrates, and DNA) caused by either increased generation and build up of the oxygen radicals (superoxide radical, hydrogen peroxide, and the hydroxyl radical), or due to the diminished removal or inadequate protection against these ubiquitously present oxygen radicals. In aerobic animals, powerful antioxidant enzymes (catalase, glutathione peroxidase, and superoxide oxidases) and radical scavengers (glutathione and vitamin E) are present to remove or scavenge these inorganic and organic oxygen radicals in cell organelles and membranes. ${ }^{2-4}$ Whenever tissues are damaged, metal complexes will be released from vacuoles, organelles, or other sequestered sites within the cell. Once released, these metal ions can then interact and propagate further lipid peroxidation. Increased MDA can be interpreted as the result of cellular membrane damage initially caused by increased formation of radicals and lipid peroxidation. But increased MDA concentrations can also result from lipid peroxidation, which only accompanies many disease processes where the initial cellular damage and release of metal complexes was elicited by toxins or other causes. ${ }^{1}$

The cerebellum appears to be more susceptible to oxidative stress than other brain structures. This observation was based upon an extensive search through the literature, from which some defined neurometabolic diseases arose where oxidative stress might be involved in the origin of damage to the cerebellum. Some well known examples associated with cerebellar atrophy are mitochondrial diseases (such as Kearns-Sayre syndrome and MERRF syndrome) or deficiency of respiratory chain enzyme complexes such as cytochrome $c$ oxidase. Other examples are Menkes' kinky hair disease, spinocerebellar degeneration due to synthetic errors of glutathione $(\gamma-$ glutamylcysteine synthetase and glutathione synthetase), or conditions with vitamin $\mathrm{E}$ depletion due to hypobetalipoproteinaemia or abetalipoproteinaemia. A common denominator in the pathogenesis of cerebellar degeneration in the above mentioned disease entities might be increased oxidative stress

In mitochondrial disorders the antioxidant enzymes and radical scavengers will fail to overcome oxidative stress because a block in the respiratory chain due to a deficient activity of one of the enzymes involved causes electron leakage and increased superoxide radical production. In patients with complex I deficiency, the subsequent release of free oxygen radical species induces grossly elevated concentrations of mitochondrial manganese superoxide dismutase. However, the antioxidant defences were not capable of preventing free oxygen radical mediated damage as reflected by multiple additional deletions of mitochondrial DNA. ${ }^{10}$

Likewise, in Menkes' kinky hair disease the disturbed copper absorption, with resultant lowered activity of the copper dependent enzyme cytochrome $c$ oxidase and cytoplasmic copper zinc superoxide dismutase, causes increased production of the superoxide radical. In conditions with glutathione or vitamin $\mathrm{E}$ depletion, the resultant impaired protection against oxygen radicals provokes increased oxidative stress. Part of the clinical correlate for these disorders is a progressive cerebellar degeneration.

It is interesting to note that MDA values were normal in healthy controls and in the majority of neurological patients with normal infratentorial structures, whereas increased MDA was found in Joubert's syndrome, pontocerebellar hypoplasia types I and II, and in many other patients with progressive cerebellar atrophy of undetermined origin. These preliminary findings suggested the possible contribution of primary or secondary oxygen radical mediated damage to early apoptosis of cerebellar progenitor cells with consequent prenatal or postnatal pontocerebellar maldevelopment or degeneration. We are currently attempting to identify the underlying cause for the observed increase of MDA in some of the patients described in this study.

1 Gutteridge JMC. Iron and oxygen: a biologically damaging mixture. Acta Paediatr Scand Suppl 1989;361:78-85.

2 Olanow CW. A radical hypothesis for neurodegeneration. Trends Neurosci 1993;16:439-44

3 Coyle JT, Puttfarcken P. Oxidative stress, glutamate and neurodegenerative disorders. Science 1993;262:689-95.

4 Halliwell B, Gutteridge JMC. Oxygen radicals and the nervous system. Trends Neurosci 1985;8:22-6.

5 Wood KA, Youle RJ. The role of free radicals and p53 in neuron apoptosis in vivo. $\mathcal{F}$ Neurol Sci 1995;15:5851-7.

6 Busciglio J, Yankner BA. Apoptosis and increased generation of reactive oxygen species in Down's syndrome tion of reactive oxygen species in Dou
neurons in vitro. Nature 1995;378:776-9.

7 Bowling AC, Beal MF. Bioenergetic and oxidative stress in Bowling AC, Beal MF. Bioenergetic and oxidative stress
neurodegenerative diseases. Life Sci 1995;56:1151-71.

8 Beal MF, Hyman BT, Koroshetz W. Do defects in mitochondrial energy metabolism underlie the pathology of neurodegenerative diseases? Trends Neurosci 1993;16:12530.

9 Wasowicz W, Nève J, Peretz A. Optimized steps in fluorimetric determination of thiobarbituric acid-reactive substances in serum: importance of extraction $\mathrm{pH}$ and influence of sample preservation and storage. Clin Chem 1993;39:2522-6.

10 Pitkänen S, Merante F, Ross McLeod D, Applegarth D, Tong T, Robinson BH. Familial cardiomyopathy with cataracts and lactic acidosis: a defect in complex I (NADHdehydrogenase) of the mitochondria respiratory chain. Pediatr Res 1996;39:513-21. 